\title{
Intelligent GA optimizer for single supply operational amplifier and MMIC low noise amplifier
}

\author{
Neoh Siew Chin ${ }^{1 *}$, Izatul Syafina Ishak ${ }^{1}, \operatorname{Lim~Wei~Jer~}^{1}$ and Arjuna Marzuki ${ }^{2}$ \\ ${ }^{1}$ School of Microelectronic Engineering, UniMAP, 02600 Pauh, Perlis, Malaysia, ${ }^{2}$ School of Electrical and Electronic Engineering, Universiti Sains Malaysia \\ *Corresponding Author: scneoh@unimap.edu.my (N.S. Chin)
}

\section{Article history :}

Received 10 January 2013

Revised 21 May 2013

Accepted 30 June 2013

Available online 1 August 2013

\section{GRAPHICAL ABSTRACT}

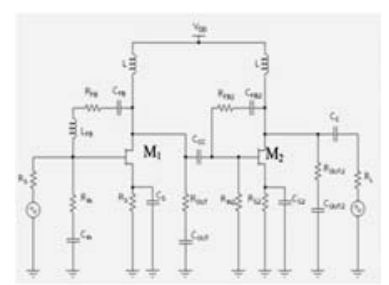

\section{ABSTRACT}

Single-Supply Operational Amplifier (Op-Amp) is important to the applications on battery-powered equipments whereas MMIC Low Noise Amplifier (LNA) is a type of integrated circuit device used in capturing operating signal in microwave frequency. This paper presents an optimization study of a SingleSupply Op-Amp and a MMIC LNA using Genetic Algorithm (GA). GA is proposed to optimize multiple input variables so as to achieve the required circuit design outputs. In Single-Supply Op-Amp, a single objective GA optimization study is conducted. Based on the single objective circuit design justification, GA is further investigated for its capability to deal with multiple design objectives in $5 \mathrm{GHz}$ MMIC LNA. In this research, the Single-Supply Op-Amp design is synthesized via LTSpice whereas the MMIC LNA design is simulated through Agilent Advanced Design System (ADS). The optimization performance of GA for both single and multi-objective circuit designs is studied and the developed multi-objective GA optimizer is further compared with ADS built-in optimizers of Gradient and Quasi-Newton respectively. From the results, GA is shown to be capable in optimizing both single objective Op-Amp and multiobjective LNA.

Keywords: Single-Supply Op-Amp, MMIC LNA, Genetic Algorithm, Optimization

(C) 2014 Penerbit UTM Press. All rights reserved http://dx.doi.org/10.11113/mjfas.v10n1.64

\section{INTRODUCTION}

Operational amplifier (op-amp) is a fundamental block building in a linear and a non-linear signal processing operations. Day by day, the op-amp becomes one of the crucial electronic devices that are used in a multiplicity of applications ranges from consumer electronics to complex commercial applications. The op-amp contains three amplifier circuits which are differential amplifier, voltage amplifier and output amplifier. These three circuits are all interconnected and contained within a single IC. The combination of these three circuits gives the key characteristics of op-amp such as high gain, high input impedance and low output impedance [1-3].

On the other hand, Monolithic Microwave Integrated Circuit (MMIC), is a type of integrated circuit (IC) device that are widely used for microwave mixing, power amplification, low noise amplification, and high frequency switching $[4,5]$. LNA is used in amplifying weak captured signals in communication systems. Many LNA designs [67] concentrated on high power supply which focuses in increasing the gain. Nowadays, Global Positioning System (GPS) is among the widely used microwave systems.
In this research, Genetic Algorithm (GA) that allows computers to solve various difficult problems with analogical genetic chromosome representations and fitness survival is used to optimize the design performance of Single-Supply Op-Amp and MMIC LNA. In term of computational intelligence, GA has been widely recognized for its robustness to deal with various optimization problems [8-9]. The research is firstly organized to investigate the capability of GA in solving single objective Single-Supply Op-Amp. The justified GA model from single objective problem is then further developed to solve multiple objectives MMIC LNA design.

\section{SINGLE OBJECTIVE SINGLE-SUPPLY OP- AMP}

The optimization of Single-Supply Op-Amp in this paper is based on the demystified Op-Amp design of Charles Kitchin [10] whereby the objective of the op-amp design is to increase the power supply rejection by considering the output gain of op-amp. Fig. 1 illustrates the schematic design of Single-Supply Op-Amp in LTSpice.

In Fig. 1, a $100 / 100 \mathrm{k} \Omega$ voltage divider for $\mathrm{R}_{\mathrm{A}}$ and $R_{B}$ with $0.1 \mu \mathrm{F}$ or acceptable capacitance value for $C_{2}$ (refer 
to Table 1) is shown. In order to achieve the goal, the circuit had been upgraded by adding some components such as $C_{2}, R_{I N}, C_{I N}$ after the voltage divider. $C_{2}$ is used to bypass the tap point on voltage divider which is restoring some ac power supply rejection. As for $\mathrm{R}_{2}$ it is used to provide a dc return path for the $\mathrm{V}_{\mathrm{s}} / 2$ references voltage and to set the circuit's ac input impedance. Table 1 shows the range of component values (input parameters) for Op-Amp performance tuning whereas Table 2 depicts the required output specification as discussed in [10]. The output gain is set in the range $20 \mathrm{~dB}$ to $40 \mathrm{~dB}$. This requirement is based on calculation of equations as follow:

$$
\begin{aligned}
& \text { Gain }=V_{\text {out }} / V_{\text {in }} \\
& \text { Gain }(d B)=20 \log \left(V_{\text {out }} / V_{\text {in }}\right)
\end{aligned}
$$

where

$$
\mathrm{V}_{\text {out }}=\mathrm{V}_{\text {in }}\left(1+\left(\mathrm{R}_{2} / \mathrm{R}_{1}\right)\right)
$$

and

$$
\mathrm{V}_{\text {in }}=15 \mathrm{~V}
$$

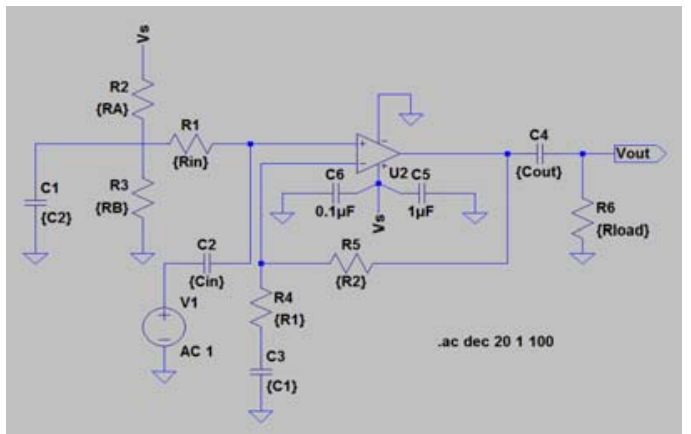

Fig. 1 Single-Supply Op-Amp Design Schematic [10] in LTSpice

Table 1 The range of component values for Single-Supply OpAmp

\begin{tabular}{|c|c|}
\hline COMPONENT & VALUES \\
\hline $\mathbf{C}_{\text {IN }}(\boldsymbol{\mu} \mathbf{F})$ & $0.1-0.3$ \\
\hline $\mathbf{R}_{\mathbf{1}}(\mathbf{k} \boldsymbol{\Omega})$ & $1.5-16.5$ \\
\hline $\mathbf{C}_{\mathbf{1}}(\boldsymbol{\mu} \mathbf{F})$ & $0.3-6.8$ \\
\hline $\mathbf{C}_{\mathbf{2}}(\boldsymbol{\mu} \mathbf{F})$ & $0.6-3.0$ \\
\hline $\mathbf{C}_{\text {OUT }}(\boldsymbol{\mu} \mathbf{F})$ & $0.05-0.2$ \\
\hline $\mathbf{R}_{\mathbf{L O A D}}(\mathbf{k} \boldsymbol{\Omega})$ & 100 \\
\hline $\mathbf{R}_{\mathbf{A}}$ and $\mathbf{R}_{\mathbf{B}}(\mathbf{k} \boldsymbol{\Omega})$ & 100 \\
\hline $\mathbf{R}_{\mathbf{2}}(\mathbf{k} \boldsymbol{\Omega})$ & 150 \\
\hline
\end{tabular}

Table 2 The required range of output gain for Single-Supply OpAmp

\begin{tabular}{|c|c|c|}
\hline \multicolumn{3}{|c|}{$\mathbf{V}_{\text {out }}=\mathbf{V}_{\text {in }}\left(\mathbf{1}+\left(\mathbf{R}_{\mathbf{2}} / \mathbf{R}_{\mathbf{1}}\right)\right)$} \\
\hline \multicolumn{3}{|c|}{$\mathbf{V}_{\text {in }}=\mathbf{1 5} \mathbf{~}$} \\
\hline $\mathbf{R}_{\mathbf{1}}(\mathbf{k} \Omega)$ & $\mathbf{R}_{\mathbf{2}}(\mathbf{k} \boldsymbol{\Omega})$ & Gain $(\mathbf{d B})$ \\
\hline 16.5 & 150 & 20 \\
\hline 7.87 & 150 & 26 \\
\hline 16.5 & 150 & 20 \\
\hline 1.5 & 150 & 40 \\
\hline
\end{tabular}

With respect to equation (1), (2), (3) and value of $\mathrm{R}_{1}$ and $R_{2}$, the required range of output gain in Table 2 is obtained. By considering the required output gain from $20 \mathrm{~dB}-40 \mathrm{~dB}$, the aim of this research is to obtain the best combination of input components that could achieve the maximum output gain of $40 \mathrm{~dB}$.

\subsection{Single objective GA optimization}

Genetic Algorithm (GA) is a family of computational models inspired by biological evolution [1115] which is traditionally used as a function optimizer. GA starts from the random population initialization of chromosomes. It allows the population to breed according to the laws of evolution, with the hoped to produce prime individual chromosomes. These chromosomes are in fact the candidate solutions of the problem. Chromosomes with good performance for the problem are expected to have a higher chance of reproduction through fitness evolution function.

Based on the fitness obtained, a key mechanism of natural selection is employed. The selected chromosomes will undergo genetic operations of crossover and mutation to produce better offspring. In crossover, genetic information of candidate solutions are exchanged and combined to produce new offspring solution. This process is intended to get the inheritance of genetic information so as to produce new candidate solutions with better fitness. As for mutation, it is a process of randomly disturbing the genetic information or flipping some genetic code to create population diversity. The genetic evolution that involves processes of selection, crossover and mutation is repeated until the termination criterion or desired optimization goal is met.

In this paper, GA is applied to optimize the SingleSupply Op-Amp developed in [10]. The overall flow of the GA operation is illustrated in Fig.2.

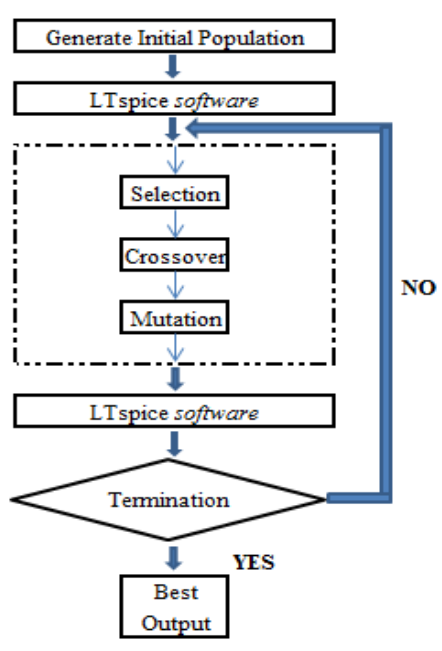

Fig.2 GA operation flow for Single-Supply Op-Amp 
i. Initial Population

In initial population, $n$ sets of individuals which represent the candidate solutions for design components of Single-Supply Op-Amp are randomly generated within the allowable range given in Table 1. The five input components to be optimized for maximum output gain are $\mathrm{C}_{\mathrm{in}}, \mathrm{R} 1$, $\mathrm{C} 1, \mathrm{C} 2$ and $\mathrm{C}_{\text {out }}$. Each generated candidate solution for these input components is then synthesized through LTspice to obtain the simulated gain.

ii. Selection, Crossover, Mutation

Based on the simulated output gain, each candidate solution is then evaluated for its fitness according to the distance of achievement towards the maximum output gain. The nearer the solution towards the design goal, the better the candidate solution is. Thus, the better the fitness, the higher the probability is for the candidate solutions to be selected for reproduction through crossover and mutation. After selection, crossover and mutation operations are then employed to produce new offspring solutions by mixing and flipping the genetic contents of the candidate solutions for genetic evolution. The new generated offspring solutions are synthesized through LTSpice for fitness evaluation after process of selection, crossover, and mutation.

iii. Termination

The termination criterion set in this research is the achievement of output gain specification. If the new generation fails to obtain output gain in the range of specification, the selection, crossover, and mutation processes will be repeated. On the contrary, the genetic evolution process will be terminated when the desired solution is found.

\subsection{Gain optimization for Single-Supply Op-Amp}

Based on the GA operation flow in Fig 2, the optimized design components of Single-Supply Op-Amp are depicted in Table 3.

Table 3 Optimize design components and best output gain for Single-Supply Op-Amp design.

\begin{tabular}{|c|c|c|}
\hline $\begin{array}{c}\text { Design } \\
\text { Components }\end{array}$ & $\begin{array}{l}\text { GA recommended } \\
\text { setting for Design } \\
\text { Components }\end{array}$ & $\begin{array}{l}\text { Output Gain } \\
\text { Obtained (dB) }\end{array}$ \\
\hline $\mathrm{C}_{\mathrm{IN}}(\boldsymbol{\mu} \mathrm{F})$ & 0.1 & \multirow{5}{*}{39.566} \\
\hline$R_{1}(k \Omega)$ & 4 & \\
\hline$C_{1}(\mu F)$ & 4 & \\
\hline$C_{2}(\mu F)$ & 1 & \\
\hline Cout $(\mu \mathrm{F})$ & 0.2 & \\
\hline
\end{tabular}

The GA recommended values for the designed components are obtained at the $10^{\text {th }}$ generation of genetic evolution cycle. This design setting produces maximum output gain of $39.566 \mathrm{~dB}$ with $6.133 \mathrm{~Hz}$ frequency. With respect to the required output specification in Table 2 , the output gain of $39.566 \mathrm{~dB}$ which is nearly $40 \mathrm{~dB}$ satisfies the aim to achieve maximum output gain for Single-Supply Op-Amp design.

\section{MULTIPLE OBJECTIVE MMIC LNA DESIGN}

Through the justification on GA's capability in optimizing single objective Single-Supply Op-Amp design, GA is further investigated for multiple objective $5 \mathrm{GHz}$ MMIC LNA design. The schematic diagram of MMIC amplifier circuit is illustrated in Fig. 3 in which a two-stage $\mathrm{RC}$ feedback amplifier is used with M1 and M2 referred as the transistors with unit gate width of $50 \mu \mathrm{m}$.

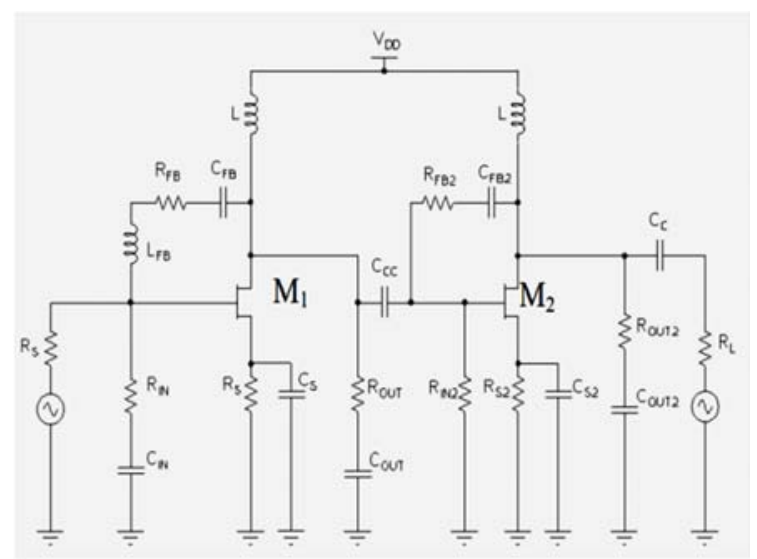

Fig. 3 MMIC low noise amplifier circuit [16]

Table 4 Design components for MMIC LNA

\begin{tabular}{|l|l|l|l|}
\hline Design Components & Symbol & From & To \\
\hline Feedback Resistor (Ohm) & $\mathrm{R}_{\mathrm{FB}}$ & 10 & 550 \\
\hline Finger Value & Finger & 2 & 10 \\
\hline Feedback Capacitor (pF) & $\mathrm{C}_{\mathrm{FB}}$ & 4 & 7 \\
\hline Output Resistor $(\mathrm{Ohm})$ & $\mathrm{R}_{\mathrm{OUT}}$ & 100 & 300 \\
\hline Output Capacitor $(\mathrm{pF})$ & $\mathrm{C}_{\mathrm{OUT}}$ & 2 & 20 \\
\hline Input Resistor $(\mathrm{Ohm})$ & $\mathrm{R}_{\mathrm{IN}}$ & 500 & 1000 \\
\hline Input Capacitor $(\mathrm{pF})$ & $\mathrm{C}_{\mathrm{IN}}$ & 2 & 20 \\
\hline Feedback Inductor $(\mathrm{nH})$ & $\mathrm{L}_{\mathrm{FB}}$ & 1 & 10 \\
\hline Coupling Capacitor (pF) & $\mathrm{C}_{\mathrm{CC}}$ & 1 & 20 \\
\hline Source Resistor (Ohm) & $\mathrm{R}_{\mathrm{S}}$ & 5 & 50 \\
\hline Source Capacitor (pF) & $\mathrm{C}_{\mathrm{S}}$ & 1 & 100 \\
\hline $\begin{array}{l}\text { Second Stage Source Resistor } \\
\text { (Ohm) }\end{array}$ & $\mathrm{R}_{\mathrm{S} 2}$ & 5 & 50 \\
\hline
\end{tabular}

Table 4 shows the range of design components to be optimized in MMIC LNA. Multiple objectives with different optimization mode are considered in this case study in which the research aims to minimize noise figure (NFmin_out) and drain current (ID), maximize power gain $(\mathrm{dB}(\mathrm{S}(2,1)))$, and satisfy the constraint limitation for circuit stability factors (mu_load and mu_source). The details of output specifications are listed in Table 5. 
Table 5 The required output specifications for MMIC LNA

\begin{tabular}{|l|l|l|l|l|}
\hline \multirow{2}{*}{ Specifications } & \multicolumn{2}{|l|}{ Value Range } & Optimization & Weight \\
\cline { 2 - 3 } & $\begin{array}{l}\text { minim } \\
\text { um }\end{array}$ & $\begin{array}{l}\text { maxim } \\
\text { um }\end{array}$ & & \\
\hline NFmin_out $(\mathrm{dB})$ & ----- & 2.5 & Minimize & 5 \\
\hline $\mathrm{dB}(\mathrm{S}(2,1))$ & 17 & 20 & Maximize & 1 \\
\hline $\mathrm{mu}$ load & 1.05 & ----- & ----- & 1 \\
\hline $\mathrm{mu}$ source & 1.05 & ----- & ----- & 1 \\
\hline ID(A) & 0.04 & 0.05 & Minimize & 10 \\
\hline
\end{tabular}

\subsection{Multiple objectives GA optimization}

In MMIC LNA optimization, the candidate solutions of design components are synthesized through ADS. Comparison study is then conducted to compare GA performance with built-in Gradient and Quasi-Newton optimizer of ADS.

Fig.4 depicts the flow of multiple objectives GA for MMIC circuit. The model starts with a random initialization of $N$ chromosomes (candidate solution) which represent the design components of MMIC LNA. By feed in the candidate solution to $\mathrm{ADS}$, the simulated output performance from ADS is then evaluated through a series of equations for fitness measurement of each specification. These fitness measurements are then further evaluated using the normalized weighted-sum approach to identify the overall fitness of a candidate solution. Equation (4-8) show the fitness function evaluation for power gain $(\mathrm{dB}(\mathrm{S}(2,1))$, noise figure (NFmin_out), drain current (ID), as well as circuit stability factors (mu load \& mu_source). A penalty value, $\alpha$ is given to the fitness function when the the required constraints are not complied.

$$
\begin{aligned}
& F_{1}=\left\{\begin{array}{l}
\alpha+(\text { NFmin_out }-2.5), \text { if NFmin_out }>2.5 \\
\alpha+\left(0.1-N F m i n \_o u t\right), \text { if NFmin_out }<0.1 \\
\text { NFmin_out }-0.1, \text { if } 0.1<\text { NFmin_out }<2.5
\end{array}\right. \\
& F_{2}=\left\{\begin{array}{l}
\alpha+(\mathrm{dB}(\mathrm{S}(2,1))-20), \text { if } \mathrm{dB}(\mathrm{S}(2,1))>20 \\
\alpha+(17-\mathrm{dB}(\mathrm{S}(2,1))), \text { if } \mathrm{dB}(\mathrm{S}(2,1))<17
\end{array}\right. \\
& 20-\mathrm{dB}(\mathrm{S}(2,1)), \text { if } 17<\mathrm{dB}(\mathrm{S}(2,1))<20 \\
& F_{3}=\left\{\begin{array}{l}
\alpha, \text { if mu_load }<1.05 \\
0, \text { if } m \text { muload }>1.05
\end{array}\right. \\
& F_{4}=\left\{\begin{array}{l}
\alpha, \text { if mu_source }<1.05 \\
0, \text { if mu_source }>1.05
\end{array}\right. \\
& F_{5}=\left\{\begin{array}{l}
\alpha+(0.04-\text { ID }), \text { if ID }<0.04 \\
\alpha+(\text { ID }-0.05), \text { if ID }>0.05 \\
\text { ID }-0.04, \text { if } 0.04<\text { ID }<0.05
\end{array}\right.
\end{aligned}
$$

As different specifications with different unit are involved, a normalized weighted function shown in equation (9) is employed to reduce possible bias of individual fitness function. The overall fitness assigned to each chromosome, $F_{\text {jtot }}$, is equivalent to the sum of the specification's fitness divided by the average fitness of the respective specification.

$$
F_{j \text { tot }}=\sum_{i=1}^{m} W_{i} \frac{F_{i}}{F_{\text {iaverage }}}
$$

where $i=1,2,3 \ldots m$ refers to the specification $i, W_{i}$ represents the preference weight given to specification $i$, and $F_{i}$ indicates the fitness of specification $i$.

The GA reproduction cycles of selection, crossover, and mutation are repeated until all the desired output specifications are achieved. Termination criterion is set based on the attainment of targeted optimization goals.

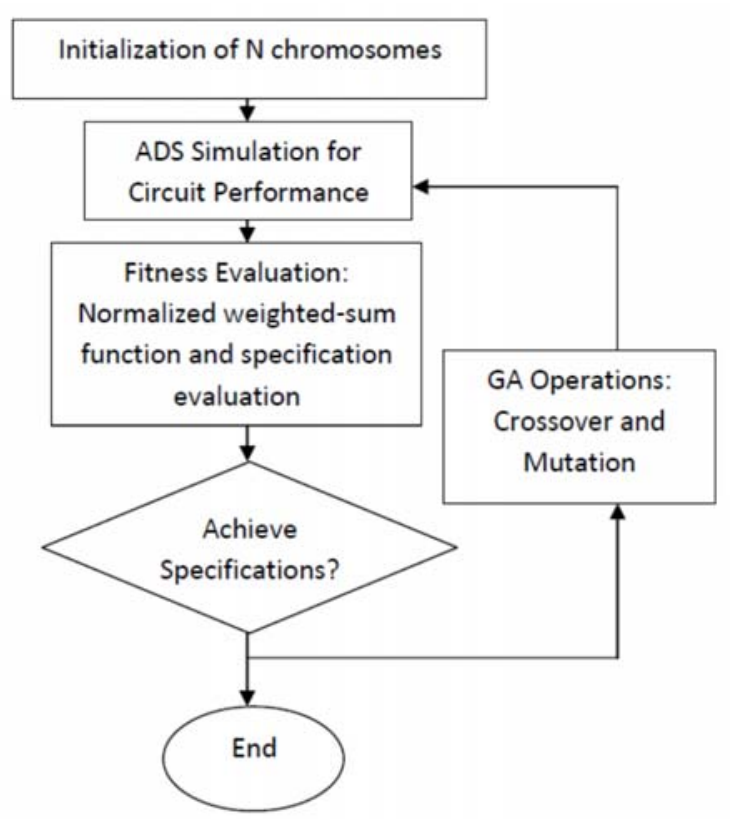

Fig. 4 Multiple objectives GA operation for MMIC LNA

\subsection{Results and discussion}

Based on the developed multiple objectives GA optimizer, the optimized design components are shown in Table 6. From the proposed setting of design components, the output specifications obtained are compared with the built-in Gradient and Quasi-Newton optimizer of ADS in Table 7.

From results in Table 7, it is observed that all GA, Gradient and Quasi-Newton optimizers fulfil the output specification ranges. Considering the preference weight for each output specifications (Table 5), although Gradient and Quasi-Newton approaches produced very good $\mathrm{dB}(\mathrm{S}(2,1))$, the achievement for ID and NFmin_out are relatively poor as compared to GA. GA generates the best NFmin_out $(19.7813 \mathrm{~dB})$ and ID $(0.0468 \mathrm{~A})$ comparing with others. Even though the produced power gain of GA is slightly lower than Gradient approach, the overall design proposed by GA still give the best total fitness as the weight ratio given to power gain is less compared to drain current and noise figure. 
Table 6 Optimized design components for MMIC LNA

\begin{tabular}{|l|l|}
\hline Design Components & GA Suggested Value \\
\hline $\mathrm{R}_{\mathrm{FB}}(\mathrm{Ohm})$ & 149 \\
\hline Finger & 10 \\
\hline $\mathrm{C}_{\mathrm{FB}}(\mathrm{pF})$ & 4 \\
\hline $\mathrm{R}_{\mathrm{Out}}(\mathrm{Ohm})$ & 123 \\
\hline $\mathrm{C}_{\mathrm{out}}(\mathrm{pF})$ & 19 \\
\hline $\mathrm{R}_{\mathrm{IN}}(\mathrm{Ohm})$ & 834 \\
\hline $\mathrm{C}_{\mathrm{IN}}(\mathrm{pF})$ & 18 \\
\hline $\mathrm{L}_{\mathrm{FB}}(\mathrm{nH})$ & 6 \\
\hline $\mathrm{C}_{\mathrm{CC}}(\mathrm{pF})$ & 10 \\
\hline $\mathrm{R}_{\mathrm{S}}(\mathrm{Ohm})$ & 47 \\
\hline $\mathrm{C}_{\mathrm{S}}(\mathrm{pF})$ & 88 \\
\hline $\mathrm{R}_{\mathrm{S} 2}(\mathrm{Ohm})$ & 32 \\
\hline
\end{tabular}

Table 7 MMIC LNA specification performance comparison of different optimizer

\begin{tabular}{|c|c|c|c|c|c|}
\hline Optimizer & $\begin{array}{l}\text { Nfmin } \\
\text { out }\end{array}$ & $\begin{array}{l}\mathrm{dB}(\mathrm{S}(2,1 \\
))\end{array}$ & mu $_{\text {load }}$ & $\begin{array}{l}\text { mu_} \\
\text { source }\end{array}$ & ID \\
\hline Gradient & 2.116 & 19.991 & 4.999 & 2.346 & 0.04996 \\
\hline Quasi-Newton & 2.167 & 19.617 & 5.19 & 2.327 & 0.04939 \\
\hline GA & 1.4911 & 19.7813 & 1.7985 & 2.1810 & 0.0468 \\
\hline
\end{tabular}

\section{CONCLUSION}

GA is a potential optimizer that could be employed to solve both single objective and multiple objective circuit designs. From the single objective Single-Supply Op-Amp case study, GA managed to produce $39.566 \mathrm{~dB}$ which is very close to the maximum power gain. Based on the single objective justification, GA's capability in solving multiple objectives is further proven in the $5 \mathrm{GHz}$ MMIC LNA design. Comparison study with Gradient and QuasiNewton methods further shows that GA is able to outperform other optimizers in optimizing design components for electronic applications.

\section{REFERENCES}

[1] Nigel P.Cook, Electronics A Complete Course (2 ${ }^{\text {nd }}$ Edition), Upper Saddle River, New Jersey Pearson Prentice Hall, 2004, 541-591.

[2] B.L David, S.S Franz and F.V Guillermo, Multimedia Tutorial on Operational Amplifier, Fundamental and Application, Department de Ingeneria Electronica, Universidad de Las Americas-Puebla, Mexico, 1998.

[3] K. H. Lundberg, Internal and External Op-Amp Compensation: A Control-Centric Tutorial, Department of Electrical Engineering and Computer Science, Massachusetts Institute of Technology, Cambridge, 2004.

[4] S. Marsh, Practical MMIC Design, Artech House, 2006.

[5] I. D. Robertson and S. Lucyszyn, IET Circuits, Devices, and System Series 13, 2001.

[6] N. Arthur, R. Tamer, and M. Yehia, Journal of Analog Integrated Circuits and Signal Processing, 55 (2008) 189.

[7] N. Trung-Kien, K. Chung-Hwan, I. Gook-Ju, Y. Moon-Su, and L. Sang-Gug, 52 (2004) 1433.

[8] M.B. Fallahpour, K.D. Hemmati, A. Pourmohammad, and A. Golmakani, Proceedings of the International Conference on Electrical Engineering and Informatics, (2011) 1.

[9] M. Gen, and R. Cheng, Genetic Algorithms and Engineering Optimization. John Wiley \& Sons Inc., New York, 2000.

[10] Charles Kitchin, Demystifying Single-Supply Op-Amp Design, Analog Devices Inc, (2002). Available website: www.ednmag.com

[11] H.L. Randy and H.E. Sue, Practical Genetic Algorithms, A WileyInterscience Publication, John Wiley \& Sons, Inc. (2004), 129.

[12] K.Rahul, V.Harsh, S.Anupam, T.Ritu, IJSCI International Journal of Computer Science, 2 (2010) 16.

[13] A.A Majida, N.I Ahmad, M.H. Zubadi., International Journal of Computer and Electrical Engineering, 2 (2010) 1793.

[14] C.Alejandro, G.Andres, L.Camilo, SAS Global Forum (2011), 149

[15] G.K. Asha, A.S Manjunath, M.A Jayaram, International Journal on Soft Computing (IJSC), 2 (2011) 15.

[16] S.C. Neoh, N. Morad, A. Marzuki, C.P. Lim, and Z.A. Aziz, Innovations in Swarm Intelligence, Springer, 2009, 121. 